\title{
Observation of Plasma Behavior in Micro-machining of Ceramics by Harmonics of Nd:YAG Laser*
}

\author{
Norihiro HAYASHI**, Yasuhiro OKAMOTO**, Yoshiyuki UNO** \\ and Tomokazu SAKAGAWA $* * *$ \\ ** Okayama University, 3-1-1 Tsushima-naka, Kita-ku, Okayama 700-8530, Japan \\ E-mail: okamoto@mech.okayama-u.ac.jp \\ ${ }^{* * *}$ Kataoka Corporation, 140 Tsukiyama-cho Kuze, Minami-ku, Kyoto 601-8203, Japan
}

\begin{abstract}
Precision micro-machining without cracks and with a narrow heat affected zone is highly required for the effective use of high-performance material. For this purpose, it is expected that the higher harmonics of Nd:YAG laser could perform the precision micro-machining by its high photon energy. However, even using harmonics of Nd:YAG laser, the heat affected zone is inevitable due to the plasma generation. In order to reduce the influence of plasma, it is important to understand the generation mechanism of plasma. Therefore, the laser induced plasma was observed by the high-speed shutter camera, and the influence of laser wavelength on machining characteristics was investigated.
\end{abstract}

Key words: Harmonics of Nd:YAG Laser, Micro-boring, Plasma, Removal Depth, High-speed Observation

\section{Introduction}

$\mathrm{AlN}$ and $\mathrm{SiC}$ have played an important role as an electronic industrial material because of its high heat conductivity and high electrical insulation ${ }^{(1)(2)}$. However, it is difficult to machine accurately by the conventional mechanical machining methods, since AlN and $\mathrm{SiC}$ as well as other ceramics are hard and brittle materials. On the other hand, the laser beam machining makes it possible to machine materials regardless of its hardness. However, in the case of fundamental wavelength of Nd:YAG laser, the heat affected zone, the dross and the thermal crack might remain on machined surfaces, since the process is mainly performed by the thermal removal ${ }^{(3)}$. Photon energy becomes higher with decreasing the wavelength of laser beam. Thus, it is considered that precision micro-machining with the low heat affect can be expected, since the material is mainly removed by photochemical reaction due to its high photon energy in the case of short wavelength laser beam, which is generated by the wavelength conversion using the nonlinear optical crystals ${ }^{(4)}$. However, even when the micro-boring of AlN and $\mathrm{SiC}$ is carried out by harmonics of Nd:YAG laser, the heat affected zone is inevitable due to the laser induced plasma. In order to reduce the influence of plasma, it is important to understand the generation mechanism of plasma.

Many investigations of laser induced plasma has been already reported ${ }^{(5)}$. However, these reports discussed phenomenon of only one kind of laser wavelength, and the influence of laser wavelength on plasma generation has not been cleared yet. Therefore, in this study, the laser induced plasma was observed by using the high-speed shutter camera in order to investigate the influence of laser wavelength on the plasma generation and machining characteristics of AlN and $\mathrm{SiC}$ by the harmonics of Nd:YAG laser, where the fundamental

*Received 9 Feb., 2010 (No. 10-0064) [DOI: 10.1299/jamdsm.4.867]

Copyright $\odot 2010$ by JSME 
wavelength $\left(\lambda_{1}=1064 \mathrm{~nm}\right)$ of $\mathrm{Nd}$ :YAG laser was converted into the second harmonic $\left(\lambda_{2}=532 \mathrm{~nm}\right)$, the third harmonic $\left(\lambda_{3}=355 \mathrm{~nm}\right)$, the fourth harmonic $\left(\lambda_{4}=266 \mathrm{~nm}\right)$ and the fifth harmonic $\left(\lambda_{5}=213 \mathrm{~nm}\right)$. Moreover, the difference of plasma generation was discussed under the atmospheric pressure and the reduced pressure conditions.

\section{Experimental method}

Figure 1 shows the schematic diagram of optical setup. The Q-switched Nd:YAG laser (QUANTEL, Brilliant) pumped by a flash lamp was used. The fundamental wavelength of $1064 \mathrm{~nm}$ in wavelength, the second harmonic of $532 \mathrm{~nm}$ in wavelength, the third harmonic of $355 \mathrm{~nm}$ in wavelength, the fourth harmonic of $266 \mathrm{~nm}$ in wavelength and the fifth harmonic of $213 \mathrm{~nm}$ in wavelength are obtained from the oscillator for the processing. The pulse repetition rate is constant at $20 \mathrm{~Hz}$, and the pulse width is $5 \mathrm{~ns}$ for every wavelength. A homogeneous intensity distribution of laser beam can provide the stable and good processing results in micro-drilling. However, the intensity distribution of laser beam from the oscillator was not good for the experimental processing due to an inhomogenation of intensity. Thus, in order to improve the intensity distribution of laser beam from the oscillator, the laser beam was expanded by 4 times using Galileo type expander, which consist of the concave lens $(\mathrm{f}=100 \mathrm{~mm})$ and the convex lens $(\mathrm{f}=400 \mathrm{~mm})$. Then, the expanded laser beam was formed by the circle mask of $5 \mathrm{~mm}$ in diameter $(\mathrm{t}=0.5 \mathrm{~mm})$. The formed laser beam was focused on the specimen surface by a lens of $100 \mathrm{~mm}$ in a focal length.

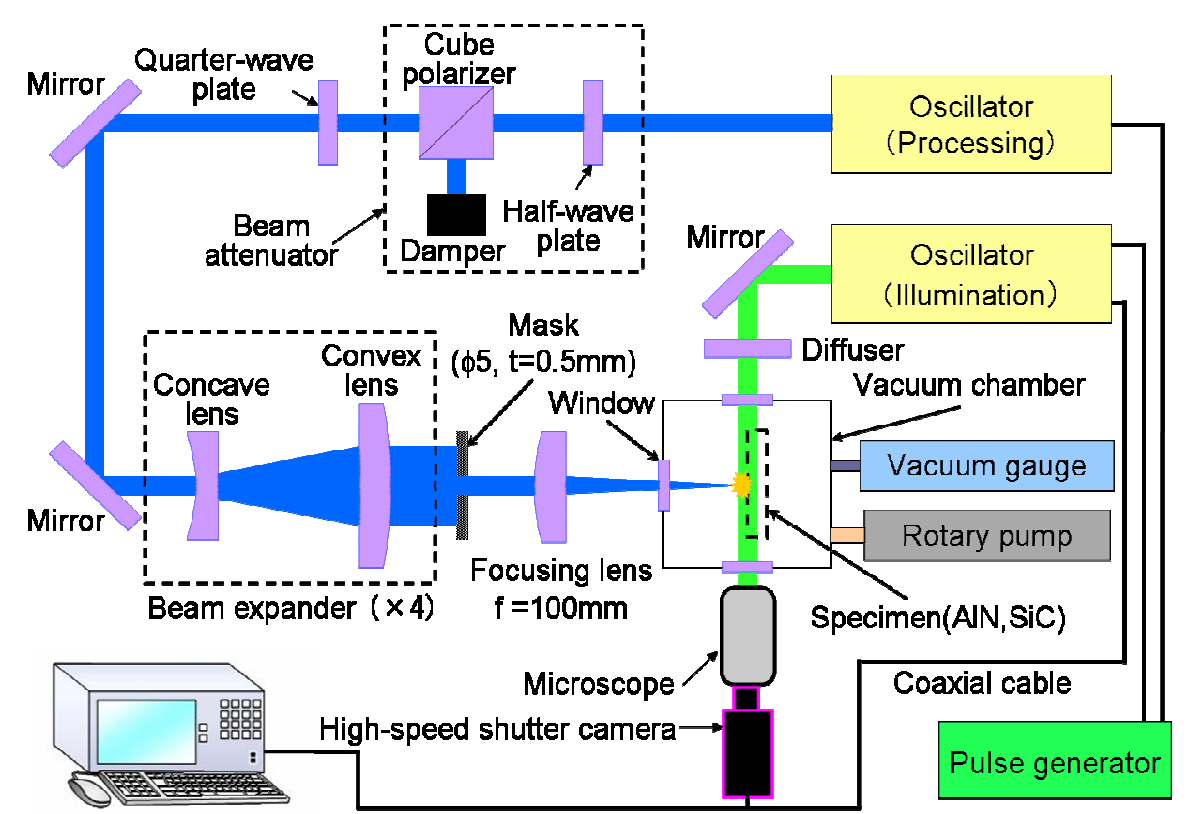

Fig. 1 Optical setup

In order to investigate the effect of the processing atmosphere between the atmospheric pressure and the reduced pressure conditions, the pressure in a vacuum chamber was controlled to $6.0 \times 10^{1} \mathrm{~Pa}$ by using the rotary pump. The high-speed shutter camera with Image Intensifier (pco.imaging, DiCAM-PRO) and zoom lens (Leica) was used in order to observe the laser induced plasma by the high-resolution time. The second harmonic of pulse width 5ns (QUANTEL, Brio) was used as the illumination light for the observation of plasma behavior to determine the position of specimen. The pulse generator (Berkeley Nucleonics Corporation, 555) was used to synchronize the laser irradiation and the shutter of camera.

Figure 2 schematically shows the laser induced plasma generated on the specimen surface. The laser beam was irradiated horizontally, and it is perpendicular to the specimen 
surface. In the measurement of laser induced plasma, the size in the laser irradiation direction was defined as a plasma height $\left(\mathrm{H}_{\mathrm{p}}\right)$ and the size perpendicular to the laser irradiation was defined as a plasma width $\left(\mathrm{W}_{\mathrm{p}}\right)$. These maximum values were recorded as the plasma height and width in this experiment.

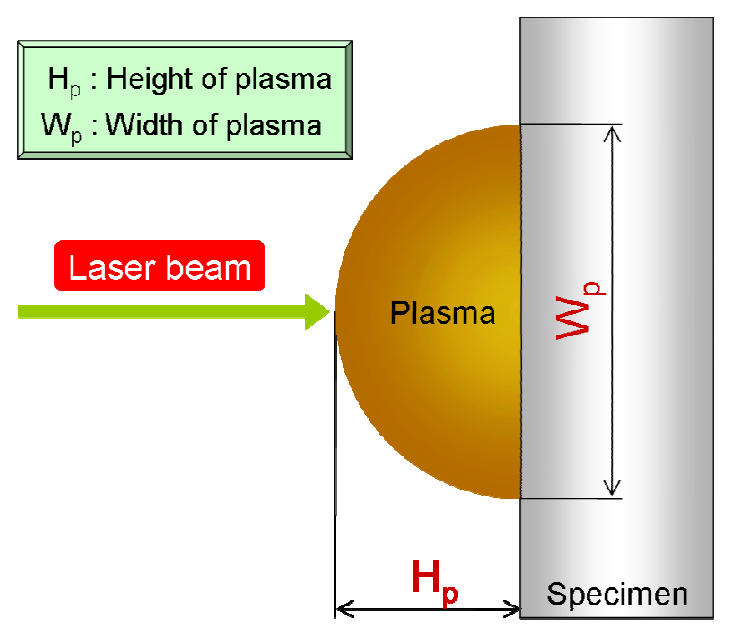

Fig. 2 Schematic illustration of laser induced plasma

\section{Results and discussion}

\subsection{Influence of laser wavelength on plasma growth}

Figure 3 shows the photographs of plasma generated on the surface AlN using various wavelengths, when the first laser shot was irradiated on the flat surface of specimen at $400 \mu \mathrm{J}$ pulse energy under the atmospheric pressure condition. The laser induced plasma was generated immediately after the irradiation for every wavelength. They grew up with increasing the time, and disappeared as an attenuation of light.

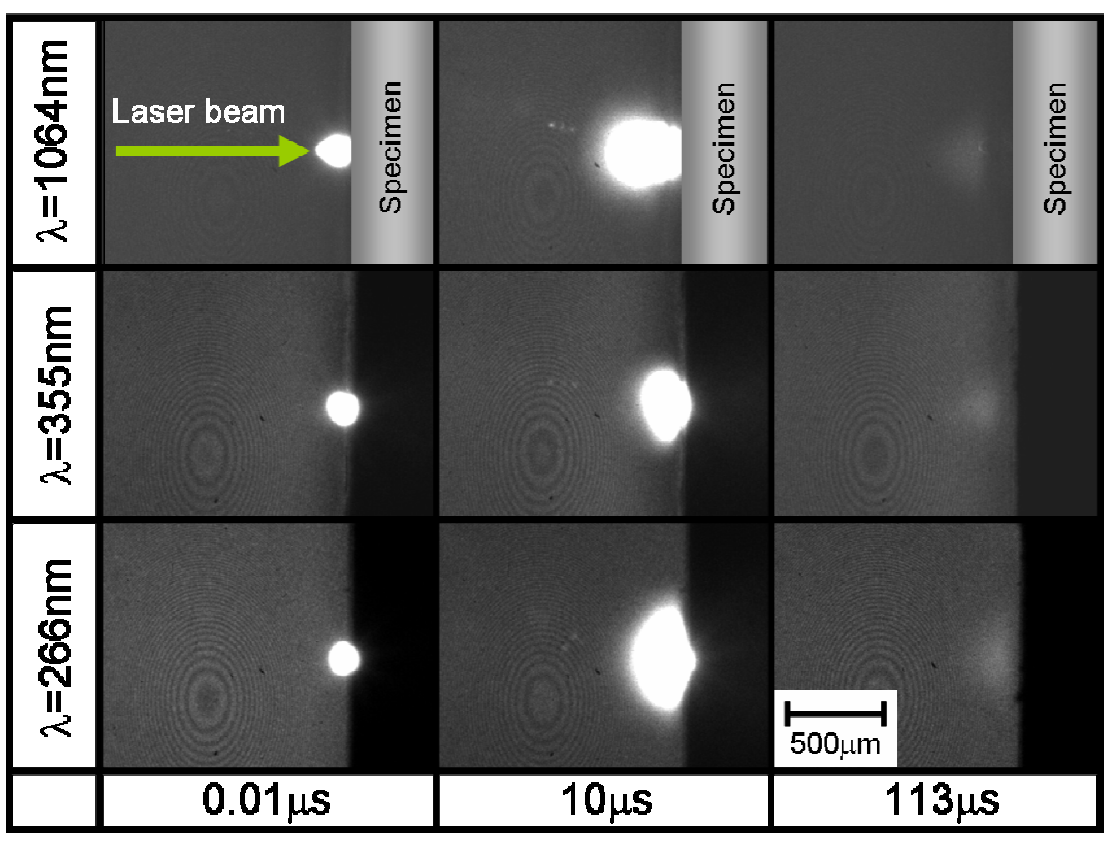

Specimen:AlN, f=100mm, E= $=400 \mu \mathrm{J} /$ pulse

Fig. 3 Photographs of plasma under the atmospheric pressure condition 
Figure 4 and Figure 5 show the change in plasma height and width measured from photographs of plasma at the first laser shot. In the height direction, the growth of fundamental wavelength was the largest. On the other hand, as for the width of plasma, the fourth harmonic is the largest.

Figure 6 shows the transmissivity of laser beam to the plasma for various wavelengths. The measurement was carried out 50ns later by controlling the delay time, when the laser beam was irradiated on the AlN surface ${ }^{(6)}$. It means that the absorption of the laser beam to the laser induced plasma increases with increasing the wavelength. Therefore, it is considered that the laser induced plasma would absorb the laser beam, and grew in the height direction for the fundamental wavelength. The difference of plasma height was remarkable at the end of laser pulse as shown in Fig.4. After the laser pulse, the plasma grew by the continuous spouting of plasma from specimens and the plasma generation of atmosphere gas, and the plasma height increased while keeping an initial difference. On the other hand, the laser beam is easy to transmit to the plasma for the higher harmonics, and the absorption of laser energy into specimens would increase. It is also reported that the absorbance is large in the higher harmonics, and the laser beam could be absorbed at the shallow depth of specimens ${ }^{(7)}$. That is why the plasma grew largely and radially in width direction in the case of higher harmonics.

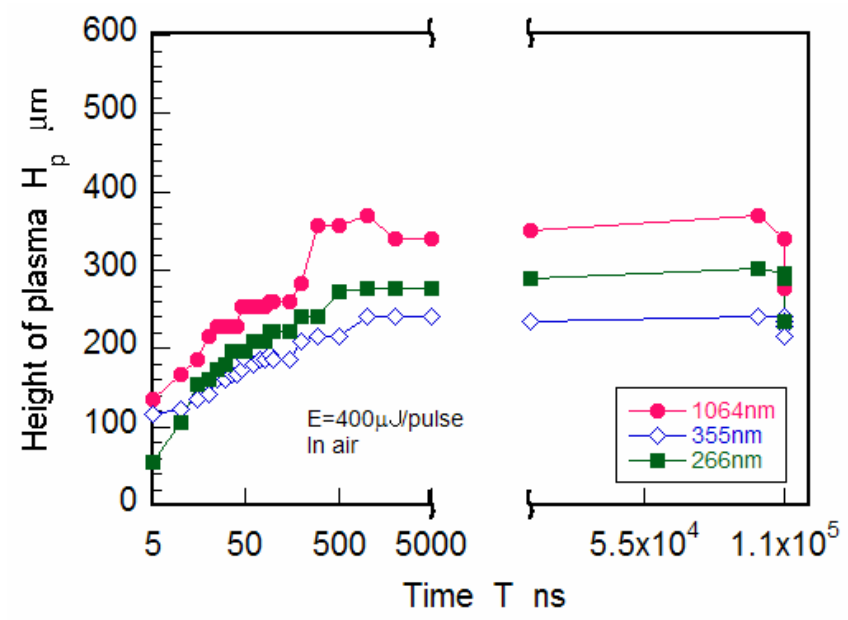

Fig. 4 Variations of plasma height with time under the atmospheric pressure condition

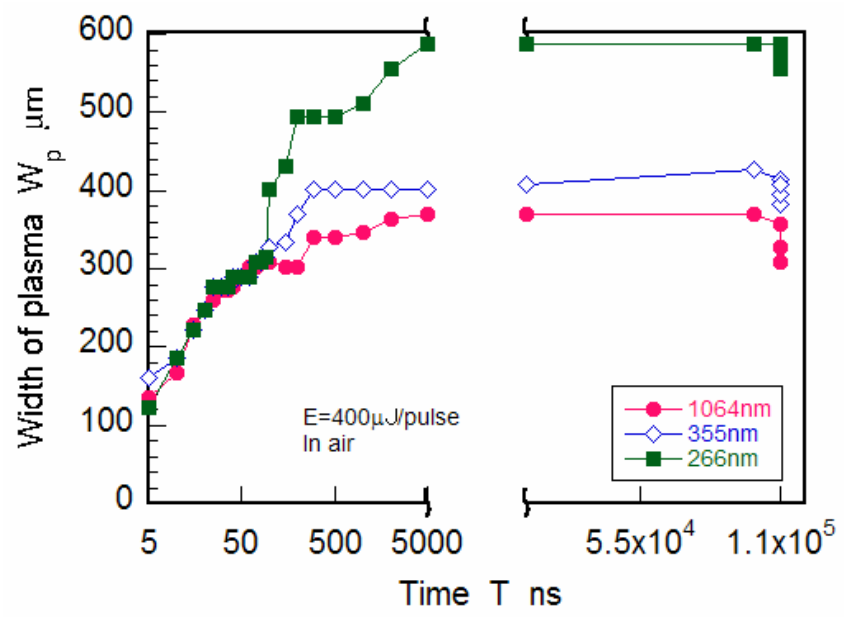

Fig. 5 Variations of plasma width with time under the atmospheric pressure condition 


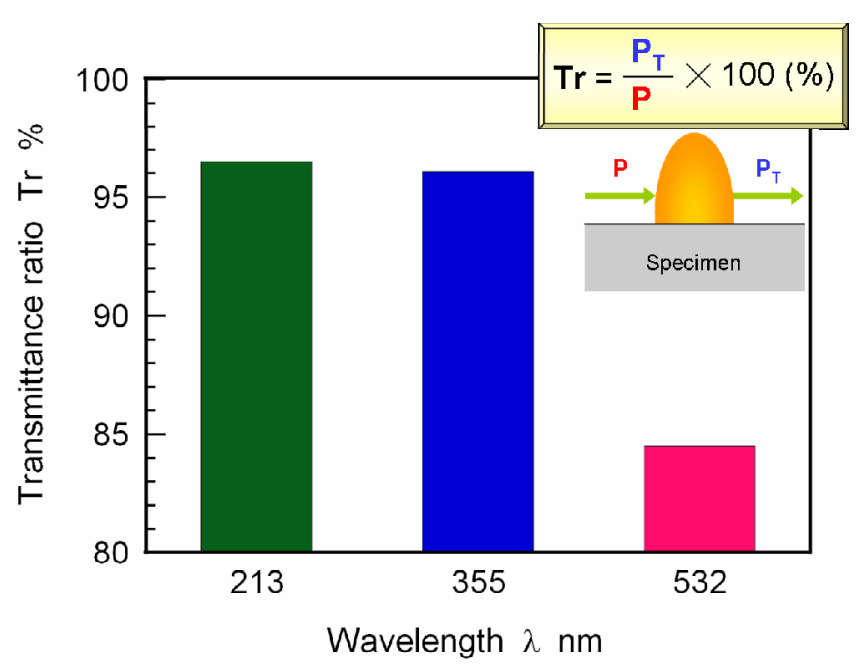

Fig. 6 Transmissivity to plasma for various laser wavelengths

Moreover, it is considered that the phenomena are not only due to the transmissivity of laser beam to the plasma generation behavior. Figure 7 shows the change in removal depth with the number of pulse and the wavelength in the case of AlN. The removal depth for 1 pulse irradiation by the fundamental wavelength is larger than that by the third and the fourth harmonic, since the material removal of AlN would be carried out by the thermal removal with cracks in the case of fundamental wavelength. The plasma which exists in the deep drilled hole can not diffuse in the width direction. Therefore, it is considered that the plasma grew largely in the height direction along the shape of drilled hole. From these results, it is revealed that the formations of laser induced plasma are greatly different by the wavelength of laser beam.

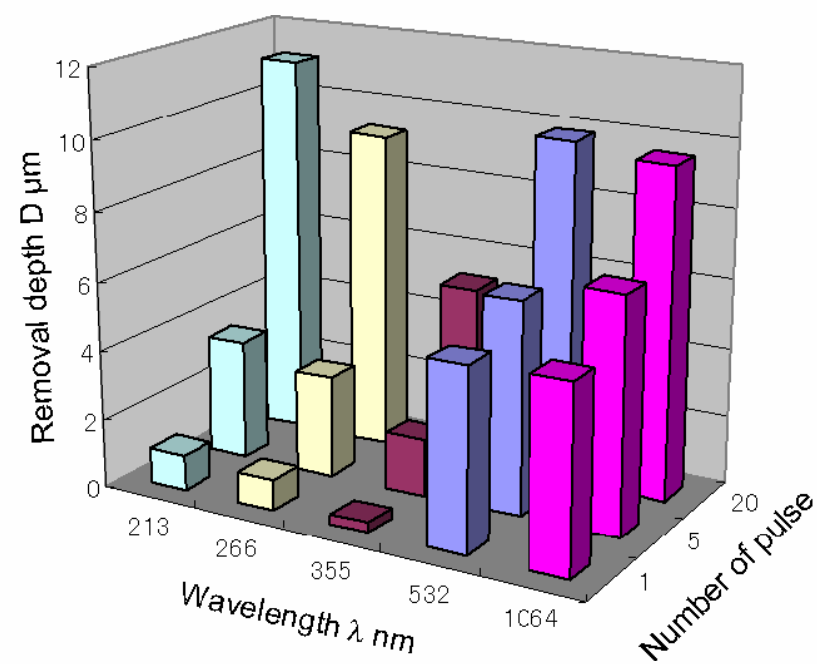

Specimen:AIN $\mathrm{E}=100 \mu \mathrm{J} /$ pulse

Fig. 7 Change in removal depth with the number of pulse and wavelength in the case of AlN

\subsection{Influence of the processing atmosphere on plasma growth}

It was reported that the removal depth of $\mathrm{AlN}$ and $\mathrm{SiC}$ under the reduced pressure condition is larger than that under the atmospheric pressure condition regardless of laser wavelength ${ }^{(8)}$. There is a possibility for the shape of plasma to influence the processing efficiency, because the laser beam is absorbed to the plasma generated on the surface of specimens as mentioned before. Therefore, the influence of processing atmosphere on plasma growth was investigated. 
Specimens were irradiated under the reduced pressure condition by the fundamental wavelength, the second harmonic and the fourth harmonic. The focusing position was set just on the surface of specimens, and the pulse energy was kept constant at $400 \mu \mathrm{J}$. The changes in plasma height and width measured from photographs are shown in Fig. 8 and Fig. 9 respectively, when the first laser shot was irradiated on the flat surface of specimen. The time of plasma growth under the reduced pressure condition is shorter than that under the atmospheric condition for every wavelength, since the rest of gas inside the vacuum chamber decreased. Therefore, the height and the width of plasma under the reduced pressure condition are smaller than that under the atmospheric pressure condition. Moreover, the differences of plasma size by the laser wavelength are remarkable in the width direction compared to that in height direction. It is considered that the growth of plasma in the height direction is influenced by the plasma spouted from specimens, and the growth of plasma in the width direction is influenced by the processing pressure.

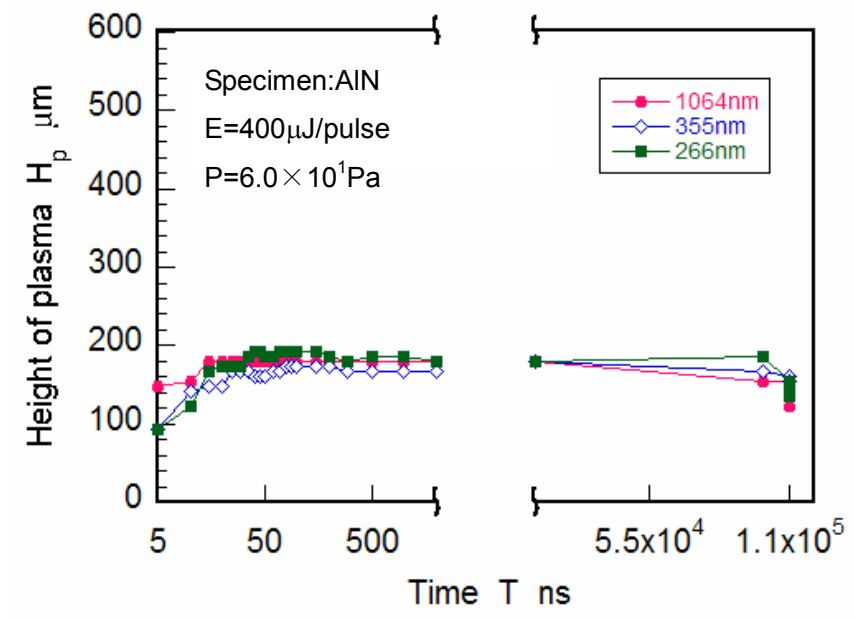

Fig. 8 Variations of plasma height with time under the reduced pressure condition

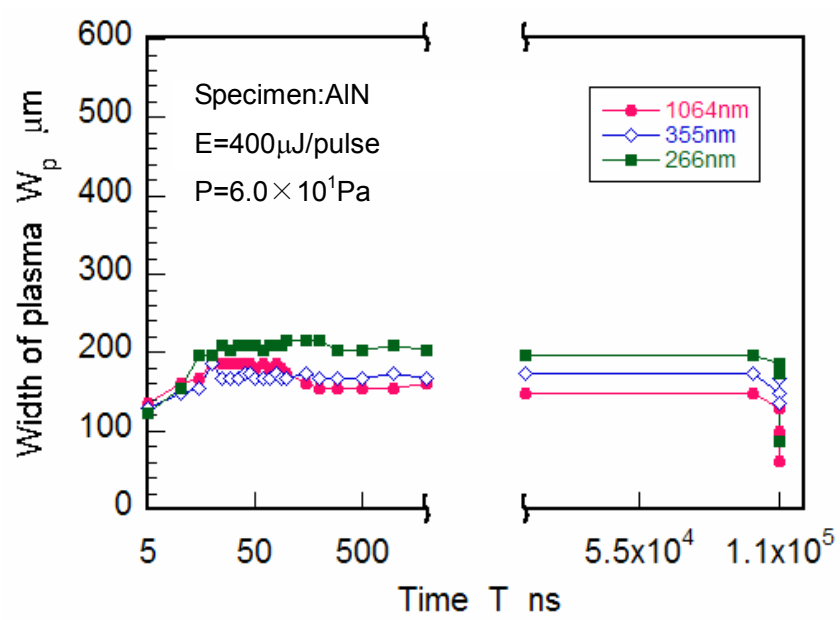

Fig. 9 Variations of plasma width with time under the reduced pressure condition

The plasma did not grow largely in the height direction for the fundamental wavelength, like under the atmospheric condition, and the difference of plasma height by the wavelength was small. It is considered that the absorbed energy of laser beam on the laser induced plasma would decrease, since the generated plasma is small under the reduced pressure condition. On the other hand, the plasma width increased with the decrease of wavelength as well as under the atmospheric condition, and the difference of plasma width 
became remarkable compared to the plasma height. From these results, it is considered that efficient processing could be expected under the reduced pressure condition, because the transmission of laser beam through the plasma, in other words, the energy absorption of laser beam on specimen increases.

\subsection{Plasma generation and surface integrity of specimens}

The surface structure around the drilled hole was greatly different between the atmospheric and the reduced pressure condition as shown in Fig. 10, where AlN and SiC were irradiated with 20 pulses under the both conditions by the fourth harmonic. The surface integrity under the reduced pressure condition was superior to that under the atmospheric pressure condition. The expanded area of plasma under the reduced pressure condition is smaller than that under the atmospheric pressure condition. Therefore, it is considered that the better surface of specimens could be obtained under the reduced pressure condition. As for $\mathrm{SiC}$, the influence of processing atmosphere became more remarkable than AIN. The dissociation energy of $\mathrm{SiC}(111.9 \mathrm{~kJ} / \mathrm{mol})$ is smaller than that of AlN $(240.5 \mathrm{~kJ} / \mathrm{mol})$. Therefore, it is expected that much more molecules of SiC might be converted to the plasma as compared to AlN. From these results, it was clear that it is important to decrease the plasma generation in order to obtain the better surface integrity of specimens around the laser irradiation points.

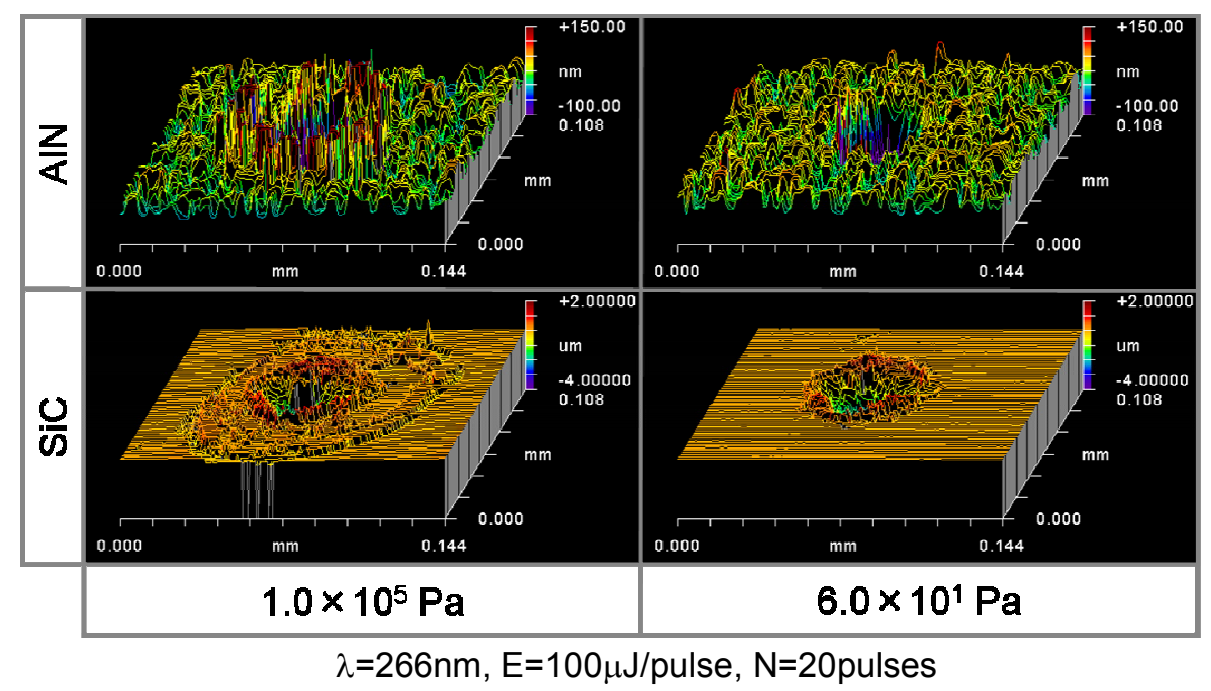

Fig. 10 3D-images of irradiated point of AIN and SiC under the atmospheric and the reduced pressure condition for wavelength $266 \mathrm{~nm}$

\section{Conclusions}

Main conclusions obtained are as follows.

1) In the case of fundamental wavelength, the plasma grows largely in the height direction by the absorption of laser beam. On the other hand, the radial growth of plasma was observed largely in the case of higher harmonics.

2) The generation of plasma under the reduced pressure condition was weaker than that under the atmospheric pressure condition. Besides, the differences of plasma generation for various laser wavelengths were small under the reduced pressure condition.

3) In order to obtain the better surface integrity of specimens, it is important to carry out the process under the reduced pressure condition. 


\section{Acknowledgements}

The authors would like to thank Ibiden Co., Ltd. for supplying expensive AlN and SiC plates. The authors are also grateful to Japan Laser Corporation for their helpful assistance in experimental setup.

\section{References}

(1) Miyajima N., New Ceramics, Chijin-shokan, (1994), p.231.

(2) O. Imanaka, Machining Handbook of Ceramics, Kensetusangyo-chousakai, (1987), p.75.

(3) Nakai S., Laser Engineering, Ohm-sha, (1999), p.91.

(4) DL Mills, Nonlinear Optics, New York springer, (1991).

(5) Matsuo Y., Journal of Plasma and Fusion Research, Vol.80, No.9 (2004), pp.777-780.

(6) Kataoka N., Okamoto Y., Uno Y. and Tanino I., Effect of Laser Wavelength on Machining Characteristics of Aluminum Nitride in Micro-boring, Proceedings of 25th International Congress on Applications of Lasers \& Electro-Optics 2006 (Laser Microprocessing Conference), (2006), pp.299-303.

(7) Liming H. and Namba Y., Spectroscopic Analysis for Machining of Inorganic Materials with the Harmonics of Nd:YAG Laser, Precision Engineering, Vol.24 (2000), pp.357-363.

(8) Okamoto Y., Sakagawa T., Nakamura H. and Uno Y., Micro-machining Characteristics of Ceramics by Harmonics of Nd:YAG Laser, Journal of Advanced Mechanical Design, Systems, and Manufacturing, Vol.2, No.4 (2008), pp.661-667. 